\title{
Ubiquity of human-induced changes in climate variability
}

Keith B. Rodgers ${ }^{1,2}$, Sun-Seon Lee ${ }^{1,2}$, Nan Rosenbloom ${ }^{3}$, Axel Timmermann ${ }^{1,2}$, Gokhan Danabasoglu ${ }^{3}$, 5 Clara Deser ${ }^{3}$, Jim Edwards ${ }^{3}$, Ji-Eun Kim ${ }^{1,2}$, Isla Simpson ${ }^{3}$, Karl Stein ${ }^{1,2}$, Malte F. Stuecker ${ }^{4}$, Ryohei Yamaguchi $^{1,2}$, Tamas Bodai ${ }^{2}$, Eui-Seok Chung, ${ }^{5}$ Lei Huang ${ }^{1,2}$, Who M. Kim ${ }^{3}$, Jean-François Lamarque ${ }^{3}$, Danica L. Lombardozzi ${ }^{3}$, William R. Wieder ${ }^{3,6}$, Stephen G. Yeager ${ }^{3}$

${ }^{1}$ Center for Climate Physics, Institute for Basic Science, Busan, South Korea

${ }^{2}$ Pusan National University, Busan, South Korea

$10{ }^{3}$ National Center for Atmospheric Research, Boulder, USA

${ }^{4}$ Department of Oceanography and International Pacific Research Center, School of Ocean and Earth Science and Technology, University of Hawai'i at Mānoa, Honolulu, HI, USA

${ }^{5}$ Korea Polar Research Institute, Incheon, South Korea

${ }^{6}$ Institute of Arctic and Alpine Research, University of Colorado, Boulder, CO, USA

15 Correspondence to: Keith B. Rodgers (krodgers@pusan.ac.kr), Axel Timmermann (axel@ibsclimate.org) 

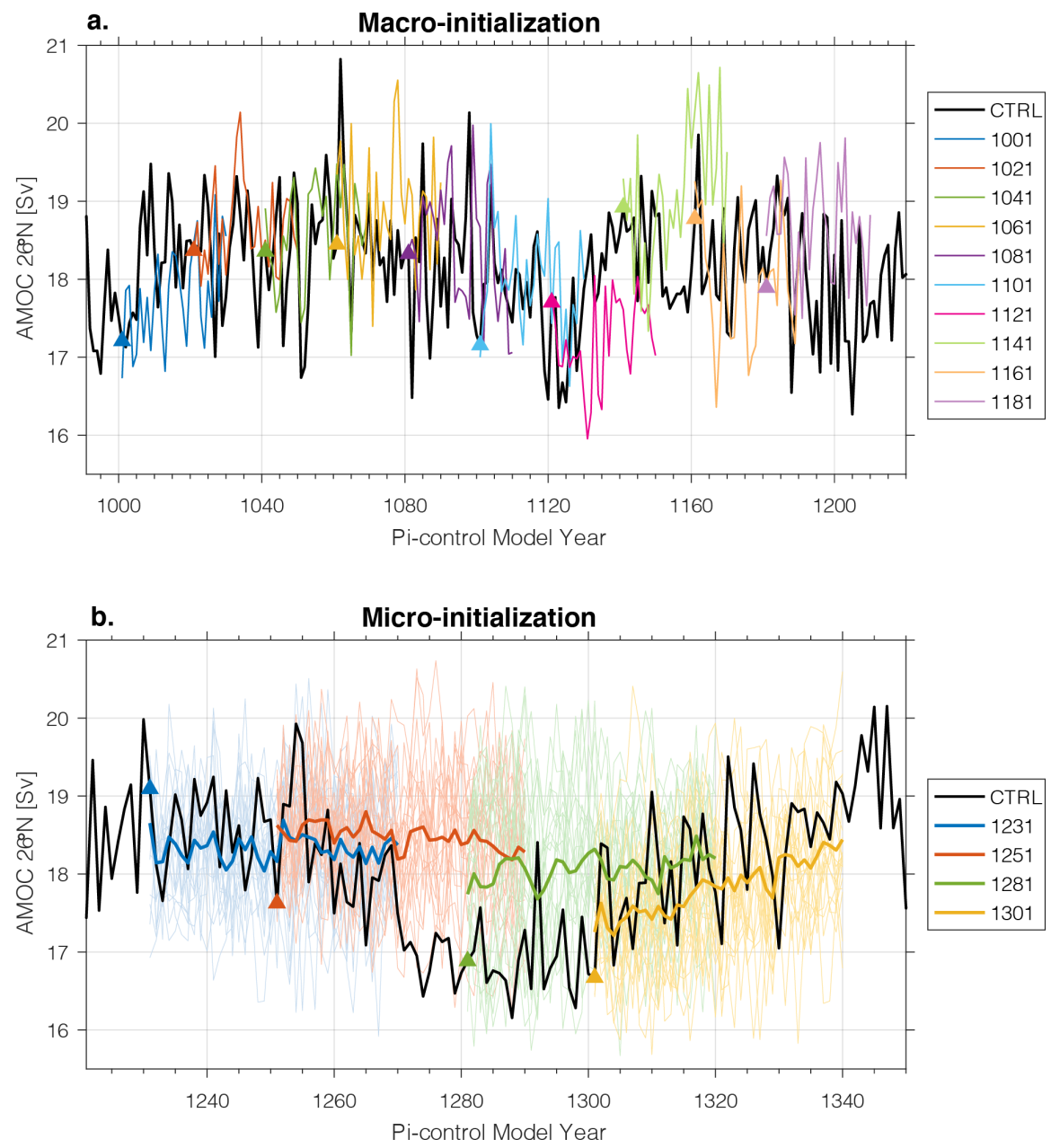

Figure S1: CESM2-LE initialization consequences for the Atlantic Meridional Overturning Circulation (AMOC) at $20 \mathbf{2 6}^{\circ} \mathbf{N}$. The AMOC transport in the pre-industrial control simulation (black line) for CESM2 is shown over two time slices corresponding to (a) 10 of the 20 macro-initializations and (b) the micro-initializations. In panel (a) the AMOC transports for the individual ensemble members are shown as solid colors, with the initiation points marked as colored triangles. In panel (b) the AMOC transports are shown in thin solid lines for individual ensemble members, and the ensemble-mean is represented as a thicker solid line. Transports are in $\mathrm{Sv}\left(10^{6} \mathrm{~m}^{3} \mathrm{~s}^{-1}\right)$. 

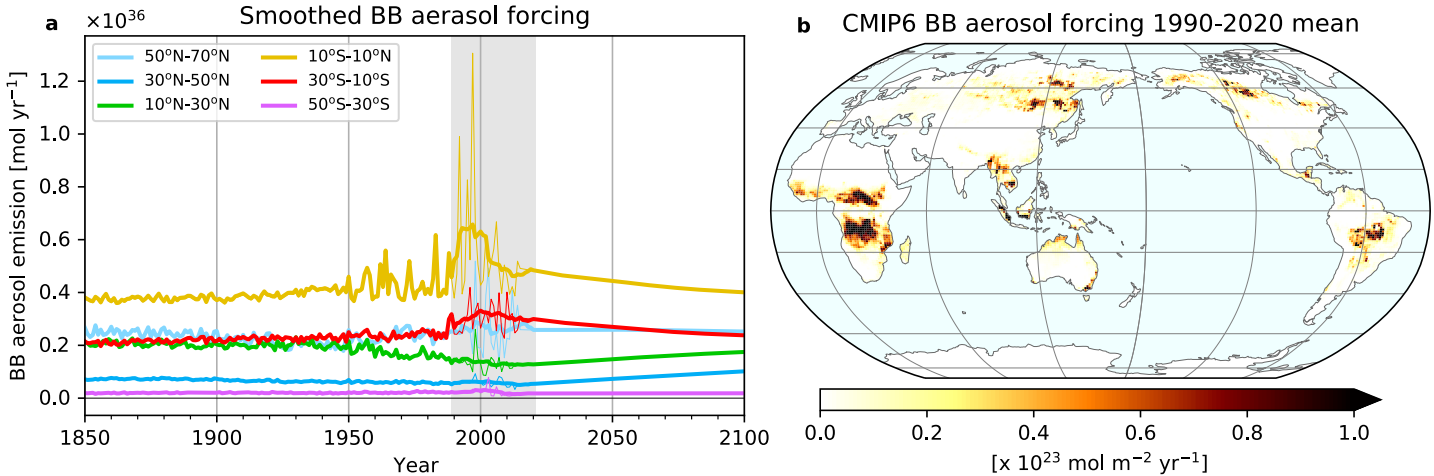

Figure S2: Biomass burning. (a) Net surface aerosol fluxes (sum of six components) associated with biomass burning are shown integrated over latitude bands, corresponding to the fluxes used for ensemble members 1-50 (color-coded light lines), and then the temporally-smoothed fields used for ensemble members 51-100 (color-coded bold lines). Smoothing is applied

30 with an 11-year running mean filter, with the averaging procedure impacting variability over the period 1990-2020, applied separately for each month to preserve the integrity of the seasonal cycle, and (b) map of mean total aerosol fluxes associated with biomass burning fluxes used for the first 50 ensemble members. The smoothing procedure applied for biomass burning is described in the Methods section. The total net surface aerosol flux is nearly conserved between the two subsets of ensembles. 
a
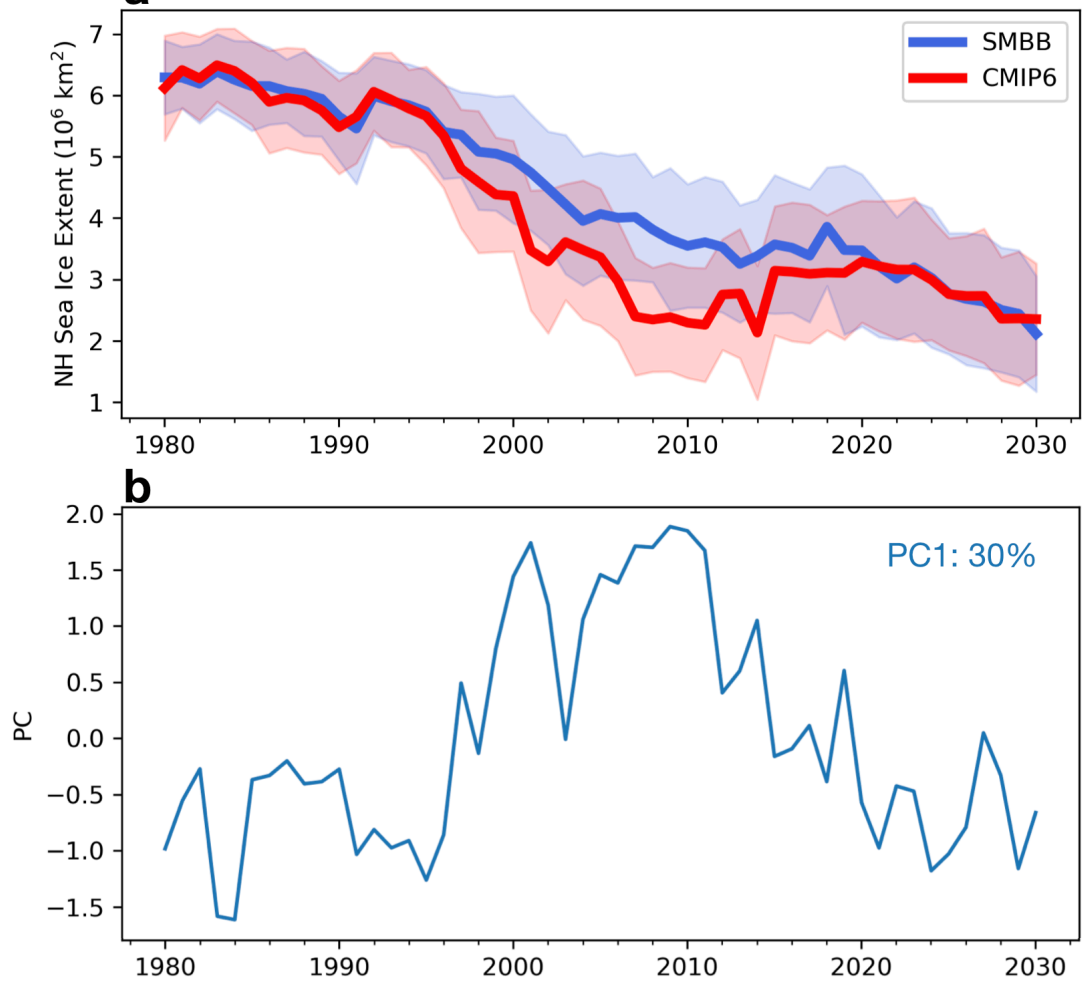

C

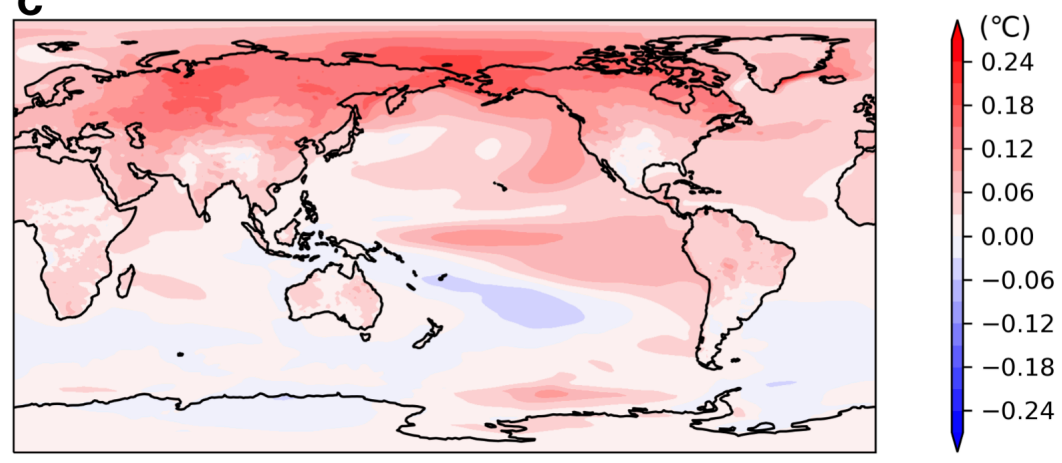

Figure S3: Impact of biomass burning perturbations on the mean state. The impact of the smoothed biomass fluxes on the mean state evolution of the model is considered for Northern Hemisphere sea-ice extent and global surface temperatures, with the 50 simulations with the original forcing (CMIP6) compared with the 50 simulations with smoothed biomass burning fluxes (SMBB). For sea ice extent in September (a) the ensemble mean for CMIP6 (thick red line) reveals a steeper decrease 40 from the early 1990s before recovering during the 2010s and eventually converging with the SMBB case (thick blue line). Lighter shading for both SMBB and CMIP5 indicates 1-SD variations. For surface temperature differences, EOF analysis has been applied to the annual mean difference in the ensemble mean with (b) the first PC and (c) the first EOF considered over 1980-2030. 

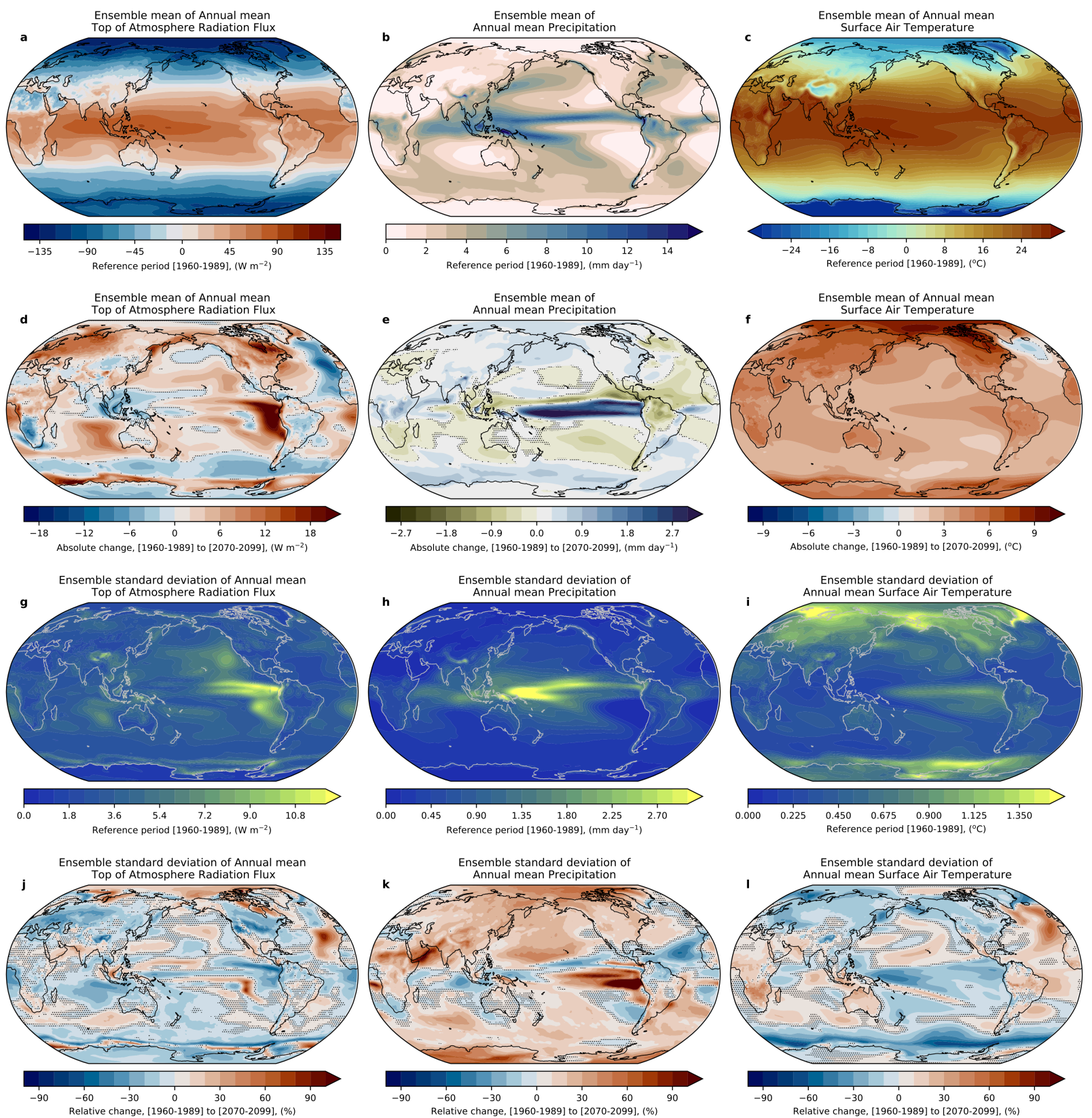

Figure S4: Patterns of change in CESM2-LE. First column: net top of the atmosphere (TOA) radiative flux; Second column: precipitation; Third column: surface temperature. First row: Mean state averaged over 1960-1989; Second Row: Change in mean state between 1960-1989 and 2070-2099; Third row: Average over 1960-1989 of snapshot (cross-ensemble) SD calculated for the annual mean of each year separately; Fourth row: Change in the amplitude of snapshot SDs between the 50 average over 1960-1989 and 2070-2099. Statistical significance of the changes (dots here reflect lack of significance $\mathrm{p} \geq 0.05$ ) was determined based on the $t$-values of the two-sample Welch's $t$-test for the equality of temporal means of the ensemble standard deviations, with the equivalent sample sizes adjusted to account for serial correlations (see Methods). Note that for mean state changes, the absolute changes are shown, whereas for standard deviation changes, relative changes are shown. Mean state changes in precipitation (e) represent the same field shown in the central map in Fig. 2, but $2 \mathrm{~m}$ reference temperature is used here whereas surface temperature is shown in Fig. 2. 

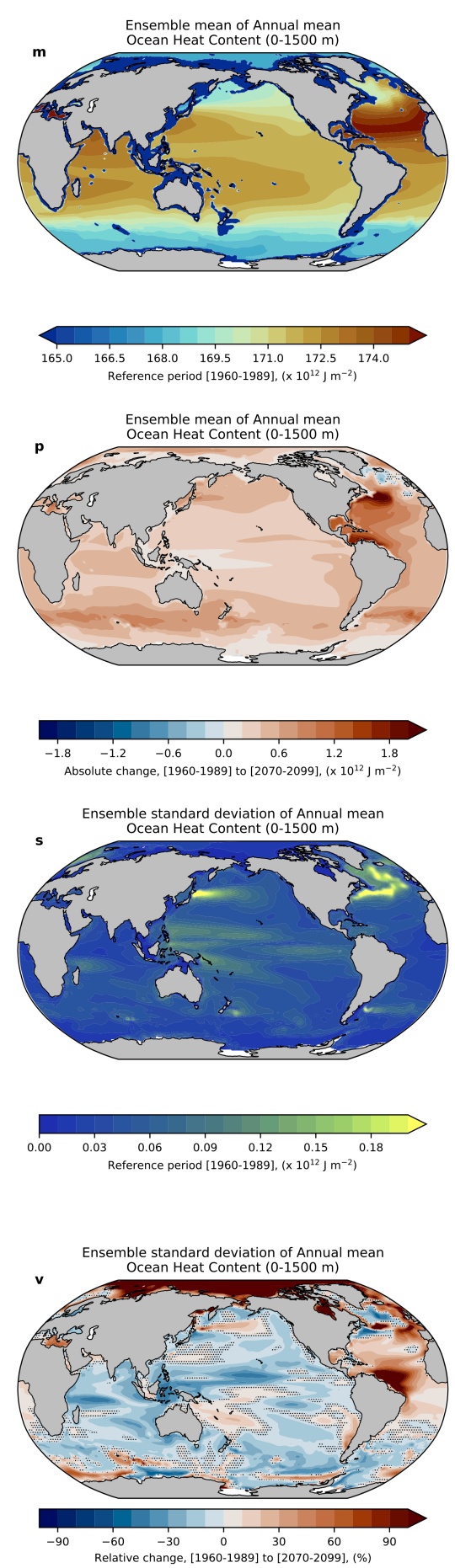
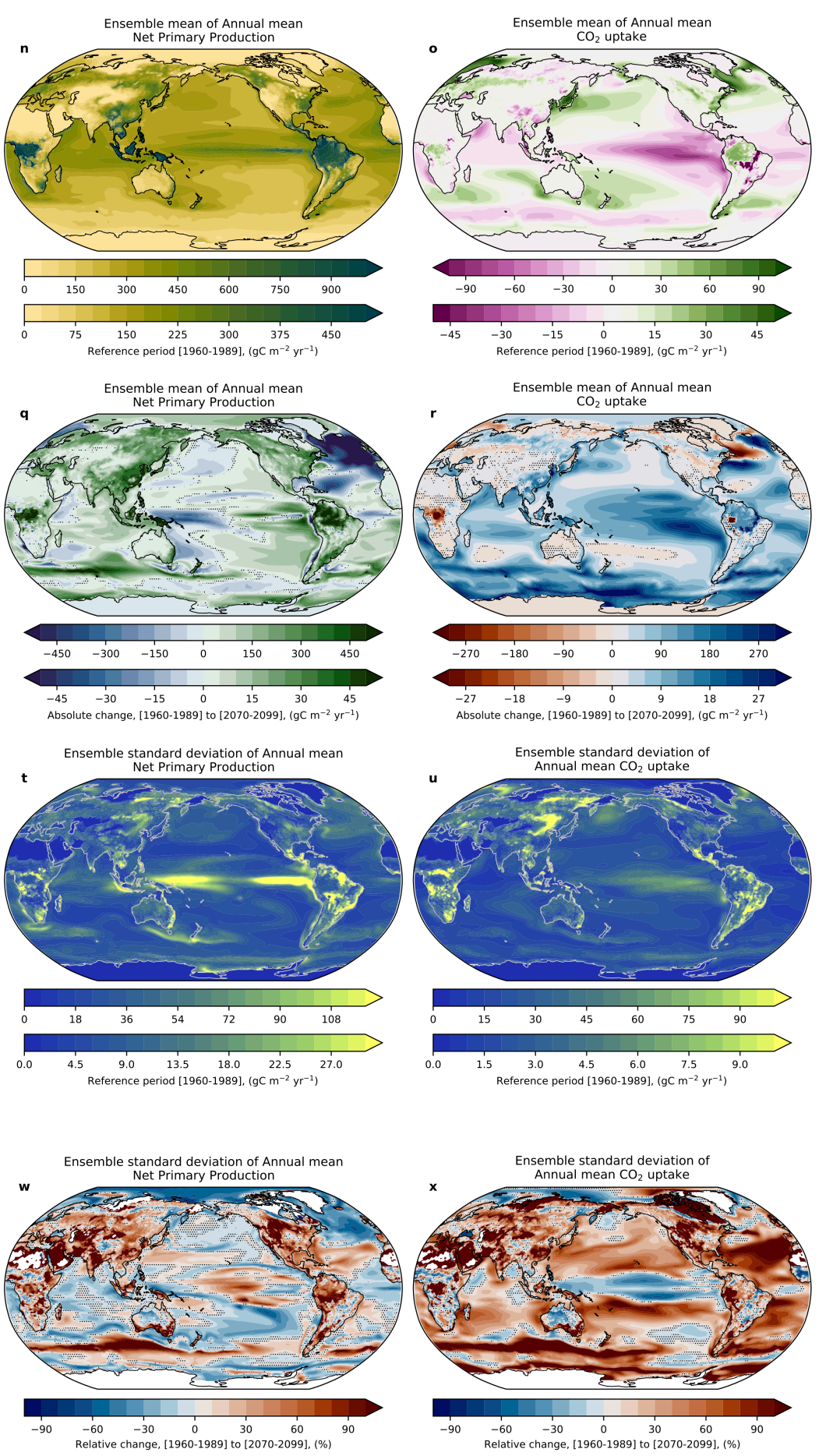

Figure S4 (continued): Patterns of change in CESM2-LE. First column: ocean heat content integrated over the upper

60 1500m; Second column: net primary production (NPP); Third column: net $\mathrm{CO}_{2}$ flux exchanged with the atmosphere. First row: Mean state averaged over 1960-1989; Second Row: Change in mean state between 1960-1989 and 2070-2099; Third row: Average over 1960-1989 of snapshot (cross-ensemble) SD calculated for the annual mean of each year separately; Fourth row: Change in the amplitude of snapshot SDs between the average over 1960-1989 and 2070-2099. Statistical significance of the changes (dots here reflect lack of significance $p \geq 0.05$ ) was determined based on the $t$-values of the two-sample Welch's

$65 t$-test for the equality of temporal means of the ensemble standard deviations, with the equivalent sample sizes adjusted to account for serial correlations (see Methods). Note that for mean state changes, the absolute changes are shown, whereas for standard deviation changes, relative changes are shown. For each panel where two underlying colorbars are shown, the upper colorbar represents quantities over land, and the lower colorbar represents quantities over the ocean. 


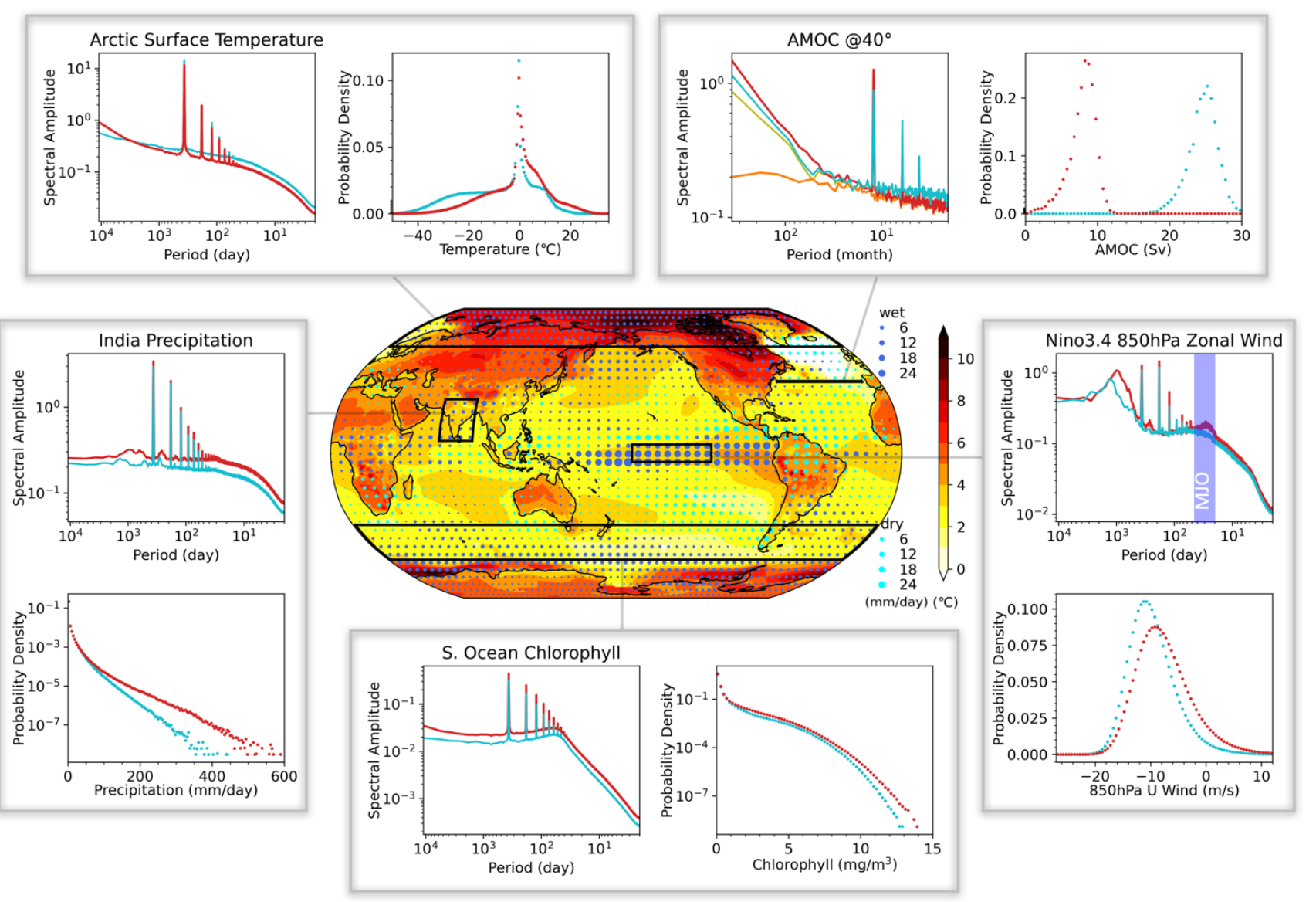

Figure S5: Changes in the Fourier amplitude spectrum of historical (1960-1989) to future (2070-2099) climate variability in CESM2-LE. The center map shows historical-to-future changes in surface temperature (shaded, ${ }^{\circ} \mathrm{C}$ ) and precipitation (mm day-1). Each pairing of panels shows the historical (cyan) and future (red) spectra and PFs for five different

75 variables over five different regions. Spectra are considered over the respective periods 1960-1989 (historical) and 2070-2099 (future), thereby including the trend, and PDFs are considered with daily data spanning 1980-1989 and 2090-2099 so as to minimize the impact of the trend. From upper-left clockwise, each pair of panels shows the historical (cyan) and future 9red) of number of daily Arctic temperature $\left(60^{\circ} \mathrm{N}-90^{\circ} \mathrm{N}\right)$, monthly Atlantic meridional overturning circulation (AMOC) at $40^{\circ} \mathrm{N}$, daily zonal wind at $850 \mathrm{hPa}$ over the Niño3.4 region $\left(5^{\circ} \mathrm{S}-5^{\circ} \mathrm{N}, 170^{\circ} \mathrm{W}-120^{\circ} \mathrm{W}\right)$, daily surface chlorophyll concentrations over

80 the Southern Ocean $\left(60^{\circ} \mathrm{S}-40^{\circ} \mathrm{S}\right)$, and daily precipitation over India $\left(7^{\circ} \mathrm{N}-30^{\circ} \mathrm{N}, 68^{\circ} \mathrm{E}-89^{\circ} \mathrm{E}\right)$. For $\mathrm{AMOC}$ variability, spectra of internal variability for the historical (olive) and future (orange) are also highlighted to show strong forced responses. The shaded area for the $850 \mathrm{hPa}$ zonal wind represents the Madden-Julian Oscillation (MJO) timescales. The Fourier spectra are calculated for timeseries at individual grid points including forced responses and internal variability and using full 30-year intervals. Subsequently, the spectra are averaged over the grid points in each region. PDFs of positive-definite variables

85 (Southern Ocean surface chlorophyll concentrations and precipitation over India) are shown with logarithmic y-axes. Sharp spectral peaks are associated with the annual cycle and its non-sinusoidal components, which generate high-order harmonics. Spectra are shown as amplitude, with the units being the same as the x-axes for the PDFs. The fields in the center panel are the same as those shown in Fig. 2. 

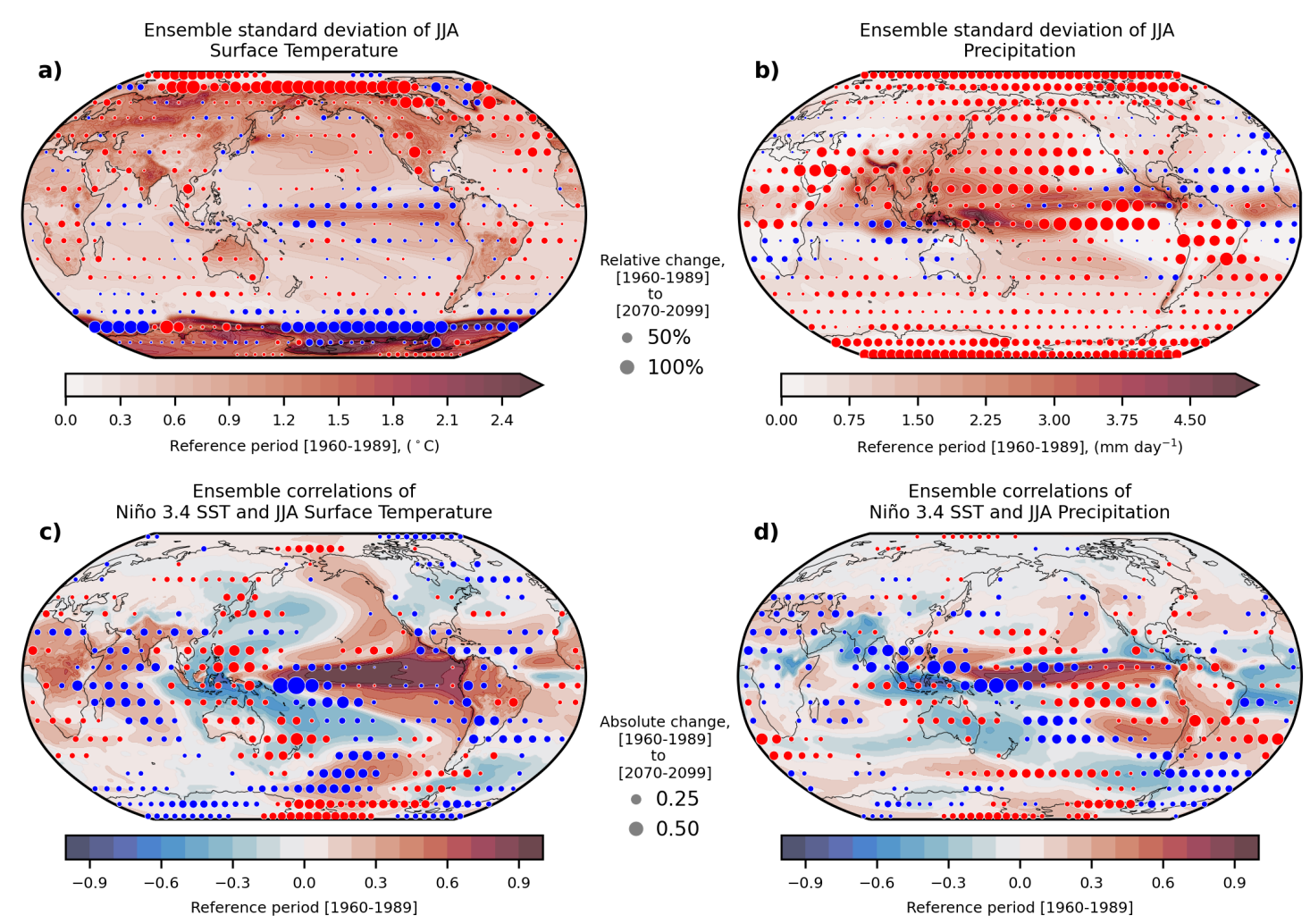

Figure S6: Changes in patterns of interannual variability and correlation coefficients of June-July-August (JJA) season Niño3.4 index with surface temperature and precipitation. (top) Colors show the time-averaged cross-ensemble standard deviation of the JJA seasonal mean surface temperature (a) and precipitation (b) for the period 1960-1989. Circles show the change in the standard deviations between 2070-2099 and 1960-1989, where insignificant change ( $p \geq 0.05)$ has been removed. (Bottom) Colors show ensemble-wise correlations of the Niño3.4 index with surface temperature (c) and precipitation (d) for JJA, averaged over the period 1960-1989. Circles show the change in correlations between 2070-2099 and 1960-1989, where statistically insignificant change $(p \geq 0.05)$ has been removed. The Niño3.4 index for ENSO is the spatial average of sea surface temperature within $5^{\circ} \mathrm{S}-5^{\circ} \mathrm{N}, 170^{\circ} \mathrm{W}-120^{\circ} \mathrm{W}$. Statistical significance of changes (circles) was determined based on the $p$ -

100 values of two-sample Student's $t$-tests for the equality of temporal means and the ensemble standard deviations (top) and of the Fisher $z$-transformed correlation coefficients. 